\title{
Demonstration of Degenerate Vector Phase-Sensitive Amplification
}

\author{
Abel Lorences Riesgo, Carl Lundström, Magnus Karlsson, Peter A. Andrekson \\ Photonics Laboratory, Department of Microtechnology and Nanoscience, Chalmers University of Technology, Gothenburg, \\ Sweden,lorences@chalmers.se
}

\begin{abstract}
The performance of a degenerate vector (dual cross-polarized pump) phase-sensitive amplifier (PSA) is characterized and compared to a degenerate scalar (dual co-polarized pump) PSA. In both schemes, we assess the gain as a function of the signal state of polarization, verifying its compliance with theory, and the phase transfer function.
\end{abstract}

\section{Introduction}

Over the last years, research on phase-sensitive fiberoptic parametric amplifiers (PS-FOPAs) has increased steadily. Unlike phase-insensitive amplifiers (PIAs), such as EDFAs, whose NF is quantum limited by $3 \mathrm{~dB}$, phase-sensitive amplifiers (PSAs) can perform noiseless amplification with $0 \mathrm{~dB}$ noise figure ${ }^{1}(\mathrm{NF})$. Moreover, all-optical regeneration of phase encoded signals based on PSAs can be achieved due to the phase squeezing provided by these amplifiers ${ }^{2}$.

Research has covered both degenerate (signal and idler at the same wavelength) and non-degenerate (signal and idler at different wavelengths) PSAs. However, experimental research has mostly focused on scalar PSAs (PSAs where all waves are copolarized), while vector PSAs (interaction between cross-polarized waves) has been studied mostly theoretically ${ }^{3}$. Whereas phase-insensitive fiber-optic parametric amplifiers (PI-FOPA) with orthogonal pumps have been studied during many years ${ }^{4}$; to the best of our knowledge, vector PS-FOPAs have not been demonstrated experimentally yet. For the first time, we demonstrate and analyze a degenerate vector PS-FOPA. As shown in Fig. 1 (a), the pumps are cross-polarized in the degenerate vector PSA scheme. The signal and idler are the two orthogonal components of the frequency degenerate wave. Hence, PS interaction occurs between the two orthogonal components of the degenerate wave. The output can be described by the following equations ${ }^{3}$ :

$$
\begin{aligned}
& S_{\text {out }}=\mu S_{\text {in }}+v I_{\text {in }}^{*} \\
& I_{\text {out }}=\mu I_{\text {in }}+v S_{\text {in }}^{*}
\end{aligned}
$$

A degenerate vector amplifier will be operating in PI mode when the degenerate wave is co-polarized to one of the pumps. Otherwise, signal and idler are present and the output depends on the phase of the input waves. The maximum PS interaction is achieved if the degenerate wave forms a $90^{\circ}$ angle (Stokes space) with the pumps. In this case, the signal (with appropriate phase) is always amplified.
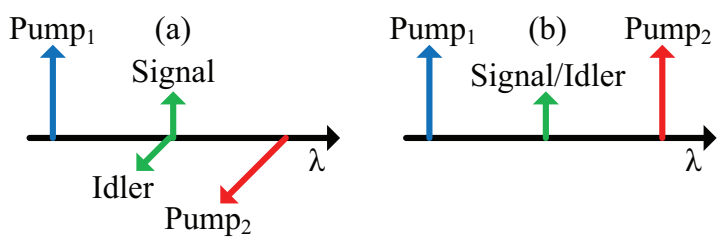

Figure 1. (a): Polarization diagram for the degenerate vector PSA. (b): Polarization diagram for the degenerate scalar PSA.

This scheme differs from the conventional degenerate scalar PSA ${ }^{2}$, Fig. 1 (b), where signal and idler are both frequency and polarization degenerate. In the degenerate scalar PSA, any signal component on the polarization orthogonal to the pumps is not affected by four-wave mixing (FWM), and its power is preserved.

Here, we compare both degenerate scalar and vector PSAs. We assess the performance of both systems with regard to the state of polarization (SOP) of the input signal. The measurements verify that these schemes have a different behaviour regarding the signal SOP. Lastly, we also characterize the phase transfer function of both schemes which demonstrates that they are indeed sensitive to the input phase.

\section{Experiment}

Firstly, we examine the gain dependence with respect to the signal SOP. The experimental setup for these measurements is depicted in Fig. 2. Two laser diodes (LDs) generate two waves at wavelengths of $1554.4 \mathrm{~nm}\left(\mathrm{LD}_{1}\right)$ and $1542.7 \mathrm{~nm}\left(\mathrm{LD}_{2}\right)$. A phase modulator (PM) modulates the phase of the wave at $1542.7 \mathrm{~nm}$ in order to avoid stimulated Brillouin scattering (SBS) in the PSA highly-nonlinear fiber $\left(\mathrm{HNLF}_{2}\right)$. Then, it is amplified by a Erbium-doped fiber amplifier (EDFA) to about $14 \mathrm{dBm}$ and filtered by an optical band-pass filter (OBPF) in order to suppress out of band amplified-spontaneous emission (ASE) noise. The $1554.4 \mathrm{~nm}$ wave is amplified by a high power EDFA to about $28 \mathrm{dBm}$. The two waves are combined by a wavelength division multiplexing (WDM) coupler before being launched to the PIFOPA consisting of $\mathrm{HNLF}_{1}$. The WDM coupler also 


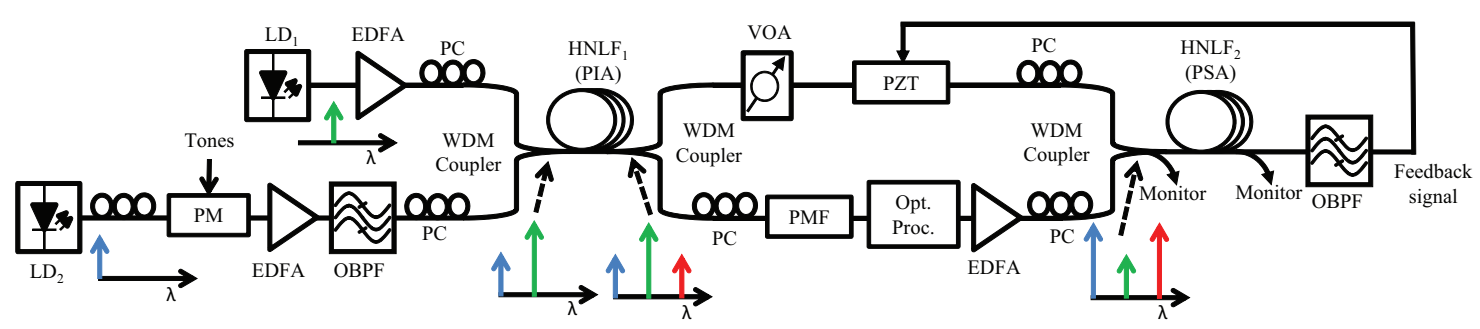

Figure 2. Block diagram of the experimental setup.

filters out of band ASE noise in the $1554.4 \mathrm{~nm}$ wave. In this PI-FOPA, or copier stage ${ }^{5}$, an idler wave at $1566.5 \mathrm{~nm}$ is generated. A WDM coupler splits these three waves into two branches. The wave at $1554.4 \mathrm{~nm}$ is directed to the upper branch before being attenuated by a variable optical attenuator (VOA). In this upper branch, a piezoelectric transducer (PZT) stabilizes the two branches. The other two waves travel through the lower branch. A polarization controller (PC) before $1 \mathrm{~m}$ of polarization-maintaining fiber (PMF) enable the possibility of obtaining cross-polarized or co-polarized waves. An optical processor (Opt. Proc.) removes the remaining power at $1554.4 \mathrm{~nm}$ and higher-order idlers created in the PI-FOPA. Then, these two waves are amplified by a high power EDFA. The three waves are combined by a WDM coupler before being launched into the PSA consisting of $\mathrm{HNLF}_{2}$. At the input of $\mathrm{HNLF}_{2}$, each pump power is about $26 \mathrm{dBm}$ and the signal power is about $-8 \mathrm{dBm}$. $\mathrm{HNLF}_{2}$ is $150 \mathrm{~m}$ long and with differential group delay (DGD) of about $0.2 \mathrm{ps}$. The monitor at the input and output of the PSA enables us to track the input and output power as well as the polarization. In order to measure the polarization, we use another optical processor so that the SOP of each wave can be measured independently.

Fig. 3 shows the gain vs. angle between signal and pump at $1542.7 \mathrm{~nm}$. The gain is measured over the degenerate wave. The angle is calculated in the Stokes space. Readers should realize that in the degenerate vector PSA, the angle between the signal and the other

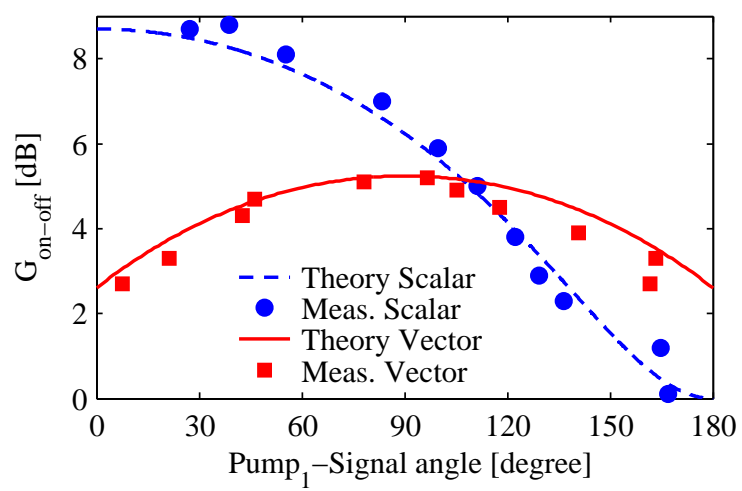

Figure 3. On-Off gain vs. angle between the polarizations of pump 1 and the signal. Lines represent the theoretical curve. Symbols represent measured data. pump is the complementary to $180^{\circ}$. The line curves represent the expected behaviour. In the degenerate vector scheme, the theoretical gain is calculated by using Eq. $(1,2)$. It can be expressed as:

$$
G_{\text {vector }}=|\mu|^{2}+|v|^{2}+2|\mu||v| \sin (\alpha) \cos (\phi)
$$

where $\alpha$ is the polarization angle between the signal and one pump, and $\phi$ is the phase difference between pumps and signal (in our case $\phi=0$ since the phaselocked loop (PLL) circuit locks the waves such that the signal is always amplified). The coefficients $\mu$ and $v$ are obtained from the maximum gain. This equation predicts a $3 \mathrm{~dB}$ difference between maximum gain (signal at $90^{\circ}$ ) and minimum gain (signal at $0^{\circ}$ and $180^{\circ}$ ) in the high-gain regime. In the degenerate scalar scheme, only the signal component on the pumps polarization is amplified phase sensitively. The gain is determined by:

$$
G_{\text {scalar }}=G(\phi) \cos ^{2}(\alpha / 2)+\sin ^{2}(\alpha / 2)
$$

where $G(\phi)$ is the conventional phase sensitive gain. As shown, the measurements comply well with theory. When the pumps are cross-polarized, the signal is always amplified. Nonetheless, when the signal SOP coincides with the SOP of either of the pumps $\left(0^{\circ}\right.$ and $\left.180^{\circ}\right)$, the amplifier is working as a PI-FOPA. A gain of about $2.7 \mathrm{~dB}$ is achieved in these cases. The maximum gain, about $5.2 \mathrm{~dB}$, is achieved when the signal is at $90^{\circ}$ with both pumps. In this case, we are operating in PS mode and tracking the phase changes is required. These measurements confirm the polarization dependence of the degenerate vector PSA scheme. As expected in the case of co-polarized pumps, the maximum gain, about $9 \mathrm{~dB}$, is higher and it occurs when the signal is co-polarized with both pumps. The predicted gain of the scalar scheme is the double in $\mathrm{dBs}$ of the vector gain when operating at perfect phase matching ${ }^{3}$. Hence, our measurements also show good agreement with the theory with regard to the gain difference between both schemes. In both cases, the achievable gain is limited by the pump power provided by the amplifier after the optical processor. Higher gain would be expected by increasing the launched pump power into $\mathrm{HNLF}_{2}$.

The setup is slightly modified in order to measure the phase transfer functions of both degenerate schemes. We totally attenuate all waves at the upper 

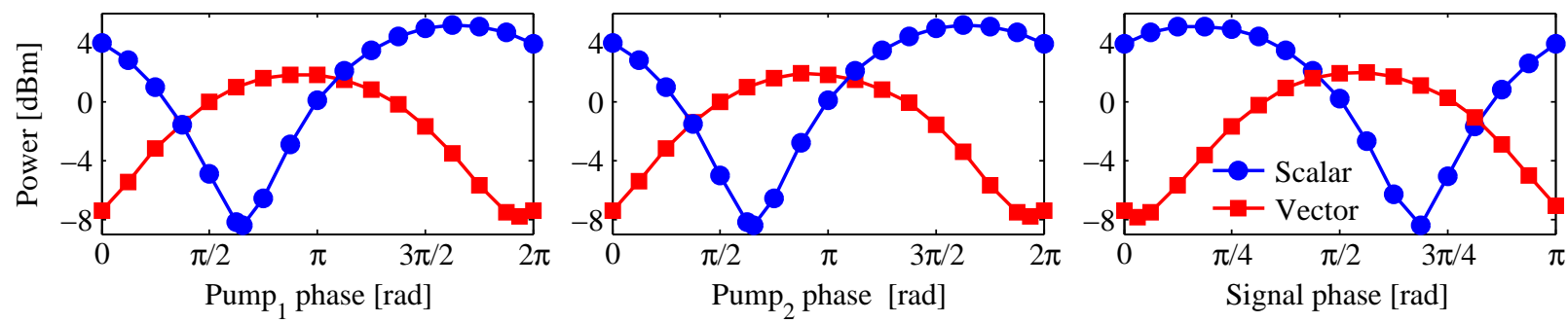

Figure 4. Measured signal power vs pump 1 phase (left), pump 2 phase (middle), signal phase (right), for the degenerate scalar (circles) and vector (squares) PS-FOPA cases.

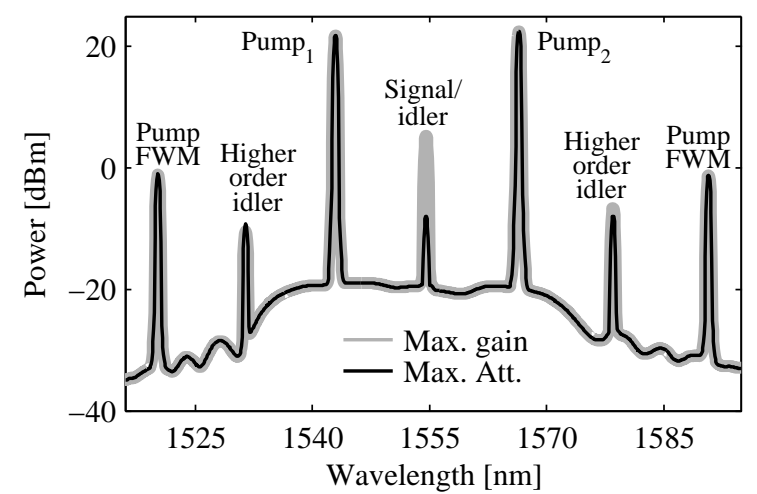

Figure 5. Output spectra at maximum gain and maximum attenuation for the degenerate scalar PS-FOPA.

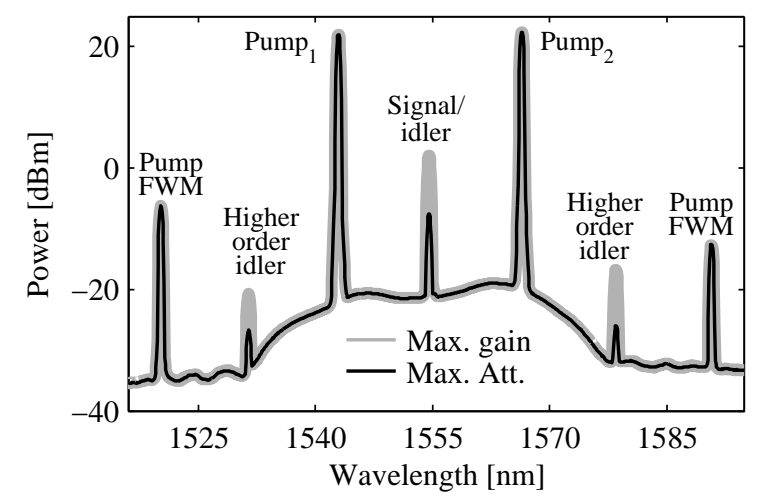

Figure 6. Output spectra at maximum gain and maximum attenuation for the degenerate vector PS-FOPA.

branch. However, the remaining signal power at the lower branch is not completely attenuated by the processor. Thus, the three waves are present in the lower branch. These three waves are launched into the $\mathrm{HNLF}_{2}$ directly after being amplified by the high power EDFA. Again, we can assess both schemes by controlling the PC before the PMF. On one hand, the three waves are co-polarized when evaluating the degenerate scalar case. On the other hand, the signal is at $90^{\circ}$ with both pumps when evaluating the degenerate vector case. Hence, both schemes could reach the maximum signal gain and maximum signal attenuation with appropriate relative phase between the three waves. The relative phase between the waves is controlled by the optical processor. Fig. 4 shows the phase transfer measurements results. As expected, both schemes have a $2 \pi \mathrm{rad}$ and $\pi \mathrm{rad}$ periodicity when sweeping the phase of any of the pumps or the signal phase respectively. Moreover, the fringes do not depend on which wave is modulated. When comparing both schemes, the swings are about 9.8 and $13 \mathrm{~dB}$ for the degenerate vector and degenerate scalar scheme respectively. Theoretically, the swing in $\mathrm{dBs}$ should be twice the maximum on-off gain in $\mathrm{dBs}$. This means that the degenerate vector scheme is working as expected with a swing very close to the expected $10.4 \mathrm{~dB}$ swing (we made sure pump powers are the same in both experiments). However, the degenerate scalar scheme achieves $5 \mathrm{~dB}$ lower than the predicted $18.2 \mathrm{~dB}$ swing. The lower swing can be explained by the presence of two additional idlers at frequencies of $2 \omega_{p}-\omega_{s}$ and $2 \omega_{s}-\omega_{p}$. As depicted in Fig. 5, these two idlers are about $10 \mathrm{~dB}$ below from the signal when maximum gain, and they have the same power as the signal when maximum attenuation. They are therefore not negligible and they are limiting the maximum swing. The presence of additional idlers can be neglected regardless of the phase of the input signal in the degenerate vector scheme. In this case, the high-order idlers have about $20 \mathrm{~dB}$ less than the signal as can be seen in the spectra for maximum gain and attenuation in Fig. 6.

\section{Conclusions}

We have demonstrated and analyzed a degenerate vector PS-FOPA for the first time. We have evaluated the gain with regard of the signal SOP and phase of the input waves. In comparison with the conventional degenerate scalar PS-FOPA, the degenerate vector PS-FOPA has lower gain. However, it is much less affected by higher-order FWM.

\section{Acknowledgments}

The authors would like to acknowledge OFS Fitel, Denmark and Sumitomo Electric Industries for providing the HNLFs.

\section{References}

[1] C.M. Caves, Phys. Rev. D, 26, 1817 (1982).

[2] R. Slavík et al., JSTQE, 18, 859 (2012).

[3] C. J. McKinstrie and S. Radic, Opt. Exp., 12, 4973 (2004).

[4] S. Radic and C.J. McKinstrie, Opt. Fiber Tech., 9, 7 (2003).

[5] Z. Tong et al., JSTQE, 18, 1016 (2012). 